\title{
A NOTE ON NATURALLY ORDERED SETS IN SEMI-METRIC SPACES
}

\author{
LOUIS F. MCAULEY
}

1. Introduction. The notion of a naturally ordered ${ }^{1}$ point set in a separable metric space and its consequences has proved to be not only useful but interesting. Whyburn [3, p. 44 (2.1)] and Zarankiewicz [4] have proved that if $G$ is any naturally ordered collection of disjoint sets in a separable metric space, then at most a countable number of elements $g$ of $G$ can contain a point which is not a condensation point ${ }^{2}$ both of the collection $P_{g}$ of all predecessors of $g$ in $G$ and the collection $F_{g}$ of all successors of $g$ in $G$. This note shows that the analogue of such a lemma holds in a hereditarily separable semimetric $^{3}$ topological space; furthermore, a theorem due to G. T. Whyburn $[3$, p. 45(2.2)] related to this lemma is also true in such a space. The proofs due to Whyburn and Zarankiewicz make use of perfect separability and do not generalize to give the above results. Thus, certain theorems which depend upon these fundamental results of Whyburn and Zarankiewicz may be established for spaces which are more general than separable metric spaces.

2. Condensation point theorem. Let $G$ denote a naturally ordered collection of disjoint sets in a semi-metric topological space $S$. Furthermore, suppose that $G^{*}$ (the logical sum of the elements of $G$ ) is hereditarily separable. First, certain lemmas analogous to ones known to be true for a separable metric space are established for a space $S$.

Presented to the Society, October 22, 1955; received by the editors September 2, 1955 and, in revised form, April 21, 1956.

${ }^{1}$ A set $N$ or collection $N$ of sets in a topological space is said to be ordered or to possess an order provided that a definition of "preceding" (indicated $<$ ) can be defined for each pair of elements which is asymmetric and transitive. Suppose that for $x \in N, P_{x}$ denotes the logical sum of all elements of $N$ preceding $x$ and $F_{x}$ the sum of all those preceded by $x$ (or following $x$ ). Then the order in $N$ is said to be a natural order if and only if for each $x \in N, \bar{P}_{x} \cdot F_{x}=P_{x} \cdot \bar{F}_{x}=0$ where $\bar{P}_{x}$ and $\bar{F}_{x}$ denote the closures of $P_{x}$ and $F_{x}$, respectively. Cf. [3, p. 41].

${ }^{2}$ Suppose that $C$ is a collection of point sets. Then a point $p$ will be called a condensation point of the collection $C$ if and only if for an open set $D$ containing $p$, there exists a subset $U$ of $D$ and an uncountable subcollection $K$ of $C$ such that if $k \in K$, then $k \cdot U$ is a point. Cf. [3, p. 44].

${ }^{3}$ If each of the letters $x$ and $y$ denotes a point of a topological $\left(T_{1}\right)$ space $S$ and there is associated with $(x, y)$ exactly one non-negative real number $d(x, y)$ called the distance from $x$ to $y$ such that (1) $d(x, y)=d(y, x),(2) d(x, y)=0$ if and only if $x=y$, and (3) each limit point of each subset $M$ of $S$ is a distance limit point of $M$ and conversely, then the space $S$ is said to be a semi-metric topological space. 
It should be noted that a hereditarily separable subset of $S$ may fail to be perfectly separable. ${ }^{4}$

The following lemma may be proved by an argument $t^{5}$ analogous to one due to R. L. Moore.

Lemma 2.1. A necessary and sufficient condition that a subset $M$ of $S$ be hereditarily separable is that every uncountable subset of $M$ have a limit point in $S$.

Lemma 2.2. An hereditarily separable uncountable subset $N$ of $S$ contains a condensation point of $N$.

Proof. Suppose that Lemma 2.2 is not true. Then there exists a number $e>0$ and an uncountable subset $N_{1}$ of $N$ such that for $p$ in $N_{1}$, there exists an $e$-neighborhood $U_{e}(p) \supset p$ where $U_{e}(p) \cdot N$ is countable. Let $\alpha=U_{e}\left(p_{1}\right), U_{e}\left(p_{2}\right), U_{e}\left(p_{3}\right), \cdots, U_{e}\left(p_{z}\right), \cdots$, denote a well ordering of the $e$-neighborhoods of the points of $N_{1}$ while $\alpha_{1}$ denotes a corresponding sequence $\left\{p_{i}\right\}$ of points of $N_{1}$. Now, there exists an uncountable sequence $\beta=\left\{p_{n_{i}}\right\}$ of points of $N_{1}$ such that (1) $p_{n_{1}}=p_{1}$ and (2) for $z>1, p_{n_{z}}$ is the first point in $\alpha_{1}$ which fails to lie in $\sum_{x \leqq n_{y}} U_{e}\left(p_{x}\right)$ for each $y<z$. By definition of $\alpha$ and $\beta$, no point of $\sum p_{n_{z}}$ is a limit point of $\sum p_{n_{z}}$. This is impossible by Lemma 2.1 since $N$ is hereditarily separable. Thus, Lemma 2.2 is true.

Lemмa 2.3. An hereditarily separable, uncountable, and naturally ordered subset $N$ of $S$ contains a point $x$ which is both a limit point of the predecessors $P_{x}$ of $x$ in $N$ and the successors $F_{x}$ of $x$ in $N$.

Proof. Suppose that $N$ contains no point $x$ such that $x \in \bar{P}_{x}$. Thus, there exists a number $e>0$ and an uncountable subset $C$ of $N$ such that for $c$ in $C, U_{e}(c) \cdot P_{c}=0$. By Lemma 2.2, $C$ contains a condensation point $p$ of $C$. Now, there exists a sequence $\left\{c_{i}\right\}$ of points of $C$ which converges to $p$ such that $p<c_{i+1}<c_{i}$ for each $i$. For some $k$, $U_{e}\left(c_{k}\right) \supset p$ contrary to the definition of $e$. It follows that there exists an uncountable subset $C_{1}$ of $C$ such that for $c$ in $C_{1}, c \in \bar{P}_{c}$; and furthermore, $C_{1} \supset x$ such that $x \in \bar{F}_{x}$. Thus, $x$ satisfies the conclusion of Lemma 2.3.

An application of these lemmas yields the following theorem.

TheOREM 2.4. At most a countable number of the elements $g$ of $G$ can contain a point which is not a condensation point both of the collection $P_{0}$

4 In a paper to appear in The Pacific Journal of Mathematics, the author shows that in a normal semi-metric topological space, hereditary separability does not imply perfect separability and hence metrizability.

5 Cf. [1] and [2, Chap. I (Theorem 46)]. Perhaps F. B. Jones first observed the truth of Lemma 2.1. 
of all predecessors of $g$ in $G$ and the collection $F_{0}$ of all successors of $g$ in $G$.

Proof. Let $N$ denote a point set such that for each $g$ in $G, g \cdot N$ is a point, and let $L$ denote the set of all points $p$ of $N$ such that $p \in \bar{P}_{p}$, $p \in \bar{F}_{p}$, and $p$ is condensation point of $N$. It follows by use of Lemmas 2.2 and 2.3 that $N-L$ is countable. For $p$ in $L$, there exist sequences $\left\{x_{i}\right\}$ and $\left\{y_{i}\right\}$ of points of $L$ which converge to $p$ such that $x_{i}<x_{i+1}$ $<p<y_{i+1}<y_{i}$ for each $i$. If $D$ is an open set containing $p$, then there exists $k$ such that $D \supset x_{k}$. Since $L$ is a subset of the naturally ordered subset $N$, there exists an open set $R \supset x_{k}$ such that $P_{p} \supset R \cdot N$. Now, $D \cdot R \cdot N$ is uncountable since $x_{k}$ is a condensation point of $N$. Similarly, it follows that $D \cdot F_{p}$ is uncountable. Hence, Theorem 2.4 is proved.

REMARK. There exist examples ${ }^{b}$ of spaces which show that the conditions that $G^{*}$ be hereditarily separable and semi-metric are both necessary for the validity of Theorem 2.4. Although these spaces are not subspaces of the number plane, their points lie in such a plane.

One of the fundamental theorems in Whyburn's theory of nonseparated cuttings as well as his cyclic element theory may now be proved for a space $S$. From Theorem 2.4 and an argument analogous to that given by Whyburn for [3, p. 45 (2.2)], the following theorem may be established. It is a generalization of Whyburn's Theorem (2.2).

Theorem 2.5. Any nonseparated collection $G$ of cuttings of a connected hereditarily separable point set $M$ in a semi-metric topological space $S$ contains a saturated subcollection $Q$ differing from $G$ by only a countable number of elements and such that each element $q$ of $Q$ is closed in $M$, irreducibly separates $M$ into just two components, and has potential order 2 in $M$ relative to $Q$.

\section{BIBLIOGRAPHY}

1. F. Burton Jones, Concerning normal and completely normal spaces, Bull. Amer. Math. Soc. vol. 43 (1937) pp. 671-677.

2. R. L. Moore, Foundations of point set theory, Amer. Math. Soc. Colloquium Publications, vol. 13, 1932.

3. G. T. Whyburn, Analytic topology, American Math. Soc. Colloquium Publications, vol. $28,1942$.

4. C. Zarankiewicz, Über die Zerschneidungspunkte der Zusammenhängend Mengen, Fund. Math. vol. 12 (1928) pp. 121-125.

University of North Carolina and

UNIVERSITY OF MARYLAND

- See Example 1.2 of the author's paper Decompositions of continua into aposyndetic continua, Trans. Amer. Math. Soc. vol. 81 (1956) pp. 74-94 and an example due to R. H. Sorgenfrey, On the topological product of paracompact spaces, Bull. Amer. Math. Soc. vol. 53 (1947) pp. 631-632. 